\title{
Review of: "High seebeck coefficient in middle- temperature thermocell with deep eutectic solvent"
}

\author{
Ani Deepthi
}

Potential competing interests: The author(s) declared that no potential competing interests exist.

This is a study which describes obtaining a high Seebeck co-efficient $\left(\mathrm{S}_{\mathrm{e}}\right)$ of a $\left[\mathrm{Fe}(\mathrm{CN})_{6}\right]^{4-/ 3-}$ thermocell, which utilizes DES ethaline as an electrolyte. The authors have proved that using the DES Choline chlorideethylene glycol has increased the efficiency of the thermocell by increasing $\mathrm{S}_{\mathrm{e}}$, the Seebeck co-efficient which in turn depends on the redox reaction entropy. The large entropy change during the reaction in thermocell is described due to interactions of the $\left[\mathrm{Fe}(\mathrm{CN})_{6}\right]^{4-/ 3-}$ with the DES. The results here add to the development of thermocells.

However, it would be nice if the following points are looked into:

1. The authors seem to have used only one type of DES namely ChCl-EG (ethaline), however the title seems to be general and so a comparative study with few other DES would be useful.

2. Will the hydrogen bond donor component and viscosity of DES affect the Se value?

3. It is well-known that the stretching frequency of a nitrile group $(\mathrm{CN})$ is sensitive to its environment. It is reported in literature earlier that a frequency of around $2224 \mathrm{~cm}^{-1}$ is observed for CN group in water and this value undergoes blue shift as the $\mathrm{H}$-bonding with $\mathrm{CN}$ increases (Zhang et. al. Phys Chem Chem Phys. 2016 Mar 14; 18(10): 7027-7034). The larger difference in FWHM value denotes the greater interaction with ChCl-EG compared to water, however the exact value of the $\mathrm{CN}$ stretch in EG is not mentioned to be compared with water and there is no literature support provided to say that $\mathrm{H}$-bonding happens more readily in $\mathrm{ChCl}-\mathrm{EG}$ compared to water.

4. What is the reason for greater concentration dependency of $S_{e}$ in ethaline thermocell than in aqueous thermocell? 